\title{
Boson Fields with Bounded Interaction Densities ${ }^{\star}$
}

\author{
RAPHAEL HÖEGH-KROHN ${ }^{\star \star}$ \\ Joseph Henry Laboratories, Princeton University, Princeton, N. J.
}

Received August 28, 1969; in revised form February 23, 1970

\begin{abstract}
We consider interaction densities of the form $V(\phi(x))$, where $\phi(x)$ is a scalar boson field and $V(\alpha)$ is a bounded real continuous function. We define the cut-off interaction by $V_{\varepsilon, r}=\int_{|x|<r} V\left(\phi_{\varepsilon}(x)\right)$, where $\phi_{\varepsilon}(x)$ is the momentum cut-off field. We prove that the scattering operator $S_{\varepsilon, r}(V)$ corresponding to the cut-off interaction exists, and we study the behavior of the scattering operator as well as the Heisenberg picture fields, as the cut-off is removed.
\end{abstract}

\section{Introduction}

In two earlier papers $[2,3]$ we studied self-interacting scalar Boson fields with interaction densities of the form $V(\phi(x))$, where $V(\alpha)$ is a bounded continuous real function. In Ref. [2] we proved that for the corresponding cut-off interaction the asymptotic limits of the fields existed. In Ref. [3] we proved that the Heisenberg picture fields existed as weak limits of the Heisenberg picture fields corresponding to the cutoff interactions. In Section 2 of this paper we prove that the Heisenberg picture fields are trivial in the sense that they are free fields. In Section 3 we prove that the scattering operator $S_{\varepsilon, r}(V)$ corresponding to the cut-off interaction exists, and we prove that the limit as $\varepsilon$ tends to zero is 1 if $r$ is small and fixed.

\section{The Heisenberg Picture Fields}

Let $\mathscr{F}$ by the Fock space of a free scalar boson field $\phi(x)$. The field operators are given in terms of the annihilation-creation operator $a^{*}$ and $a$ by

$$
\phi(x)=2^{-\frac{1}{2}}(2 \pi)^{-\frac{3}{2}} \int_{R^{3}} e^{i p x}\left(a(p)+a^{*}(-p)\right) d p .
$$

* This research partially sponsored by the Air Force Office of Scientific Research under Contract AF 49(638)1545.

$\star \star$ At leave from Mathematical Institute, Oslo University. 
For annihilation-creation operator we use the Lorentz invariant commutation relations

$$
\left[a(p), a^{*}\left(p^{\prime}\right)\right]=\omega(p)^{-1} \delta\left(p-p^{\prime}\right),
$$

where $\omega(p)=\left(m^{2}+p^{2}\right)^{\frac{1}{2}}$. We assume that the mass $m$ of the free field is strictly positive. Let $\mathscr{H}=L_{2}\left(R^{3}, \omega^{-1}(p) d p\right)$, then $\mathscr{H}$ carries in a natural way an irreducible representation of the inhomogeneous Lorentz group. Since $\mathscr{F}$ is the direct sum of the symmetric tensor products of $\mathscr{H}, \mathscr{F}$ also carries a representation of the inhomogeneous Lorentz group. For $h \in \mathscr{H}$ we set $a^{*}(h)=\int a^{*}(p) h(p) d p$, where $a^{*}$ stands for $a^{*}$ or $a$. Then $a^{*}(h)$ are closed operators with domain containing $D_{0}$, the domain of the free energy operator $H_{0}$. Moreover $a(\bar{h})$ and $a^{*}(h)$ are adjoint operators with the same domain of definition, hence $a^{*}(h)+a(\bar{h})$ are self-adjoint with domain containing $D_{0}$. The commutation relations for $a^{*}(h)$ may be written

$$
\left[a(\bar{h}), a^{*}(g)\right]=(h, g) .
$$

Let $g$ be in $C_{0}^{\infty}\left(R^{3}\right)$, such that $g \geqq 0, g(x)=g(-x), \int g(x) d x=1$ and $g$ has support in the open sphere of radius 1 and center at the origin in $R^{3}$. Let $g_{\varepsilon}(x)=\varepsilon^{-1} g\left(\varepsilon^{-1} x\right)$, then $g_{\varepsilon}$ has support in the open sphere of radius $\varepsilon$, and $g_{\varepsilon}$ tends to Dirac's $\delta$-distribution as $\varepsilon$ tends to zero. Define now the cut-off field operators by

$$
\phi_{\varepsilon}(x)=\int g_{\varepsilon}(x-y) \phi(y) d y .
$$

By what is said above the annihilation-creation operators, we see that $\phi_{\varepsilon}(x)$ is a self-adjoint operator with domain containing $D_{0}$, the domain of $\mathrm{H}_{0}$.

Let $V(\alpha)$ be a bounded continuous real function. Then $V\left(\phi_{\varepsilon}(x)\right)$ is a bounded self-adjoint operator, such that $\left\|V\left(\phi_{\varepsilon}(x)\right)\right\| \leqq\|V\|_{\infty}=\sup _{\alpha}|V(\alpha)|$. Since $V\left(\phi_{\varepsilon}(x)\right)=U(-x) V\left(\phi_{\varepsilon}(0)\right) U(x)$, where $U(x)$ is a strongly continuous unitary group, we see that $V\left(\phi_{\varepsilon}(x)\right)$ is strongly continuous in $x$. Therefore we may define

$$
V_{\varepsilon, r}=\int_{|x|<r} V\left(\phi_{\varepsilon}(x)\right) d x,
$$

where the integral is a strong integral. $V_{\varepsilon, r}$ is then a bounded self-adjoint operator, and we have the following $\varepsilon$-independent estimate for its norm.

$$
\left\|V_{\varepsilon, r}\right\| \leqq \frac{4 \pi}{3} r^{3}\|V\|_{\infty} .
$$

The cut-off energy operator is defined by

$$
H_{\varepsilon, r}=H_{0}+V_{\varepsilon, r} .
$$


Since $V_{\varepsilon, r}$ is a bounded self-adjoint operator we get that $H_{\varepsilon, r}$ is a selfadjoint with the same domain $D_{0}$ as $H_{0}$.

Let $h$ be real and in $L_{2}\left(R^{3}\right)$, we then define the free Heisenberg picture field and the Heisenberg picture field for the cut-off interaction by

$$
\begin{aligned}
\phi^{t}(h) & =e^{-i t H_{0}} \phi(h) e^{i t H_{0}} \\
\phi_{\varepsilon, r, t}(h) & =e^{-i t H_{\varepsilon, r}} \phi(h) e^{i t H_{\varepsilon, r}}
\end{aligned}
$$

where $\phi(h)=\int \phi(x) h(x) d x$. Since $e^{i t H_{0}}$ and $e^{i t H_{\varepsilon, r}}$ leave $D_{0}$ invariant we see that the operators defined above are self-adjoint operators with domain containing $D_{0}$.

Lemma 1. Let $V(\alpha)$ be a real function which is the Fourier transform of an $L_{1}$-function $\hat{V}(s)$. Then

$$
V_{\varepsilon, r}=\int_{|x| \leqq r} V\left(\phi_{\varepsilon}(x)\right) d x=\int_{|x| \leqq r} \int d s \hat{V}(s) e^{i s \phi_{\varepsilon}(x)},
$$

and $V_{\varepsilon, r}$ converge weakly to zero as $\varepsilon$ tends to zero.

Proof. Let $\Omega$ be the Fock vacuum. Then $\Omega$ is in the domain of $e^{a^{*}(h)}=\sum_{n=0}^{\infty} \frac{1}{n !}\left(a^{*}(h)\right)^{n}$ for $h$ in $\mathscr{H}$. To see this we have only to compute the norm of $\sum_{n=0}^{\infty} \frac{1}{n !}\left(a^{*}(h)\right)^{n} \Omega$, and the computation gives us $\left\|e^{a^{*}(h)} \Omega\right\|^{2}$ $=e^{\|h\|^{2}}$, which is finite. Moreover the set of vectors $e^{a^{*}(h)} \Omega$, with $h$ real and in $\mathscr{H}$ spends a dense set in $\mathscr{F}$. This is easieast seen by taking the strong derivative of $e^{a^{*}\left(s_{1} h_{1}+\cdots+s_{n} h_{n}\right)} \Omega$ with respect to $s_{1}, \ldots, s_{n}$ at zero. By doing so we get $a^{*}\left(h_{1}\right) \ldots a^{*}\left(h_{n}\right) \Omega$, and these vectors we know spends a dense set in $\mathscr{F}$ for $h_{1}, \ldots, h_{n}$ real and in $\mathscr{H}$. Since the strong partial derivative is formed by taking a strong limit of linear combinations of vectors of the form $e^{a^{*}\left(s_{1} h_{1}+\cdots+s_{m} h_{m}\right)} \Omega$, we conclude that the vectors $e^{a^{*}(h)} \Omega$ with $h$ real and in $\mathscr{H}$ spends a dense set.

The spectral theory gives us the identity

$$
V_{\varepsilon, r}=\int_{|x| \leqq r} d x \int d s \hat{V}(s) e^{i s \phi_{\varepsilon}(x)} .
$$

Since $\hat{V}$ is in $L_{1}$, we get by Lebesgue's lemma on dominated convergence that it is enough to prove that $e^{i s \phi_{\varepsilon}(x)}$ converge weakly to zero for almost all $s$ and $x$. Using that $e^{i s \phi_{\varepsilon}(x)}=U(-x) e^{i s \phi_{\varepsilon}(0)} U(x)$, it is enough to prove that $e^{i s \phi_{\varepsilon}(0)}$ tends weakly to zero for almost all $s$. By uniform boundedness it is therefore enough to prove that

$$
\left(e^{a^{*}\left(h_{1}\right)} \Omega, e^{i s \phi_{\varepsilon}(0)} e^{a^{*}\left(h_{2}\right)} \Omega\right)
$$

converge to zero for almost all $s$ when $h_{1}$ and $h_{2}$ are real and in $\mathscr{H}$. 
From the definition of $\phi_{\varepsilon}(0)$ we see that $\phi_{\varepsilon}(0)=a\left(h_{\varepsilon}\right)+\mathrm{a}^{*}\left(h_{\varepsilon}\right)$, where $h_{\varepsilon}=2^{-\frac{1}{2}}(2 \pi)^{-\frac{3}{2}} \hat{g}_{\varepsilon}$. The commutation relations then gives us

$$
e^{i s \phi_{\varepsilon}(0)}=e^{-\frac{1}{2} s^{2}\left\|h_{\varepsilon}\right\|^{2}} e^{i s a^{*}\left(h_{\varepsilon}\right)} e^{i s a\left(h_{\varepsilon}\right)} .
$$

Hence (2.6) is equal to

$$
\begin{aligned}
& e^{-\frac{1}{2} s^{2}\left\|h_{\varepsilon}\right\|^{2}}\left(e^{-i s a\left(h_{\varepsilon}\right)} e^{a^{*}\left(h_{1}\right)} \Omega, e^{i s a\left(h_{\varepsilon}\right)} e^{a^{*}(h)} \Omega\right)
\end{aligned}
$$

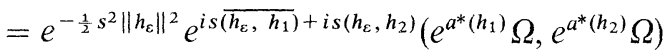

$$
\begin{aligned}
& =e^{-\frac{1}{2} s^{2}\left\|h_{\varepsilon}\right\|^{2}} e^{i s\left(\overline{\left.h_{\varepsilon}, h_{1}\right)}+i s\left(h_{\varepsilon}, h_{2}\right)\right.} e^{\left(h_{1}, h_{2}\right)} .
\end{aligned}
$$

Since $h_{\varepsilon}$ as well as $h_{1}$ and $h_{2}$ are all real, we get that $\overline{\left(h_{\varepsilon}, h_{1}\right)}$ and $\left(h_{\varepsilon}, h_{2}\right)$ are both real. Therefore the absolute value of (2.6) is bounded by $e^{\frac{1}{2} s^{2}\left|h_{\varepsilon}\right|^{2}} e^{\left(h_{1} \cdot h_{2}\right)}$. It is easy to see that $\left|h_{\varepsilon}\right|^{2}$ tends to infinity as tends to zero, and this gives us that (2.6) tends to zero for almost all $s$. This proves the lemma.

Lemma 2. Let $V(\alpha)$ be a real continuous function which tends to zero at infinity. Then

$$
V_{\varepsilon, r}=\int_{|x| \leqq r} V\left(\phi_{\varepsilon}(x)\right) d x
$$

converge weakly to zero as $\varepsilon$ tends to zero.

Proof. It is well known that a real continuous function which tends to zero at infinity may be uniformly approximated by the Fourier transform of $L_{1}$-functions. Hence for any $\delta>0$ we can find a $\tilde{V}(\alpha)$ which is the Fourier transform of an $L_{1}$-function such that $\|V-\tilde{V}\|_{\infty}<\delta$. Let $\tilde{V}_{\varepsilon, r}=\int_{|x|<r} \tilde{V}\left(\phi_{\varepsilon}(x)\right) d x$. From the spectral theory of self-adjoint operators we know that $\left\|\tilde{V}\left(\phi_{\varepsilon}(x)\right)-V\left(\phi_{\varepsilon}(x)\right)\right\| \leqq\|V-\tilde{V}\|_{\infty}<\delta$. This gives us that $\left\|\tilde{V}_{\varepsilon, r}-V_{\varepsilon, r}\right\| \leqq \frac{4}{3} \pi r^{3} \delta$. Let $\psi_{1}$ and $\psi_{2}$ be vectors of unite length in $\mathscr{F}$. Then

$$
\left|\left(\psi_{1}, V_{\varepsilon, r} \psi_{2}\right)\right| \leqq \frac{4}{3} \pi r^{3} \delta+\left|\left(\psi_{1}, \tilde{V}_{\varepsilon, r} \psi_{2}\right)\right| .
$$

By Lemma 1 the last term on the right hand side tends to zero as $\varepsilon$ tends to zero. This gives us that

$$
\varlimsup_{\varepsilon \rightarrow 0}\left|\left(\psi_{1}, V_{\varepsilon, r} \psi_{2}\right)\right|<\frac{4}{3} \pi r^{3} \delta .
$$

Since $\delta$ is arbitrary the lemma is proved.

Theorem 1. Let $V(\alpha)$ be a continuous real function which tends to zero at infinity. Then $V_{\varepsilon, r}=\int_{|x| \leqq r} V\left(\phi_{\varepsilon}(x)\right) d x$ converge strongly to zero as $\varepsilon$ tends to zero, for all values of $r$.

Proof. Since any real continuous function $V(\alpha)$ which tends to zero at infinity, may be written as a difference $V(\alpha)=V^{+}(\alpha)-V^{-}(\alpha)$ of two positive continuous functions which both tend to zero at infinity, we see that $V_{\varepsilon, r}=V_{\varepsilon, r}^{+}-V_{\varepsilon, r}^{-}$, where $V_{\varepsilon, r}^{ \pm}=\int_{|x|<r} V^{ \pm}(\phi(x)) d x$. Hence it is sufficient 
to prove that $V_{\varepsilon, r}$ tends strongly to zero for $V(\alpha)$ positive. But if $V(\alpha)$ is positive then $V_{\varepsilon, r}$ is a positive operator with a unique square root $V_{\varepsilon, r}^{\frac{1}{2}}$. Since $\left\|V_{\varepsilon, r}^{\frac{1}{2}} \psi\right\|^{2}=\left(\psi, V_{\varepsilon, r}^{\frac{1}{2}} \psi\right)$ we get by Lemma 2 that $V_{\varepsilon, r}^{\frac{1}{2}}$ converge strongly to zero as $\varepsilon$ tends to zero. From (2.5) it follows that $V_{\varepsilon, r}^{\frac{1}{2}}$ is a norm bounded uniformly in $\varepsilon$. Hence $V_{\varepsilon, r}=V_{\varepsilon, r}^{\frac{1}{2}} . V_{\varepsilon, r}^{\frac{1}{2}}$ is the product of two strongly convergent and uniformly bounded operators. Therefore we conclude that $V_{\varepsilon, r}$ converge strongly to the product of the limits which is zero. This proves the theorem.

Since $D_{0}$ is the domain of $H_{0}$ as well as $H_{\varepsilon, r}$ we know that both $e^{i t H_{\varepsilon, r}}$ and $e^{i t H_{0}}$ leaves $D_{0}$ invariant. $D_{0}$ is a Hilbert space with its natural norm $\left\|\left(H_{0}+1\right) \psi\right\|$. Since $V_{\varepsilon, r}$ is bounded this norm is equivalent to the norm $\left\|\left(H_{\varepsilon, r}+b\right) \psi\right\|$ for $b$ large enough. Therefore besides being unitary groups on $\mathscr{F}, e^{i t H_{\varepsilon, r}}$ and $e^{i t H_{0}}$ are semigroups on $D_{0}$. As semigroups on $D_{0}$ they are strongly continuous in $t$, and uniformly bounded in $t$. Moreover, $e^{i t H_{\varepsilon, r}}$ as an operator on $D_{0}$ is uniformly bounded in $t$ and $\varepsilon$. To see the strong continuity let $\psi$ be in $D_{0}$, then

$$
\begin{aligned}
\left\|\left(H_{0}+1\right)\left(e^{i t H_{\varepsilon, r}} \psi-\psi\right)\right\| & \leqq a\left\|\left(H_{\varepsilon, r}+b\right)\left(e^{i t H_{\varepsilon, r}} \psi-\psi\right)\right\| \\
& \leqq a\left\|\left(e^{i t H_{\varepsilon, r}}-1\right)\left(H_{\varepsilon, r}+b\right) \psi\right\|
\end{aligned}
$$

which tends to zero by the strong continuity of $e^{i t H_{\varepsilon, r}}$ on $\mathscr{F}$. The strong continuity of $e^{i t H_{0}}$ and the uniform boundedness in $t$ of $e^{i t H_{\varepsilon, r}}$ and $e^{i t H_{0}}$ is proved in the same way. To see that $e^{i t H_{\varepsilon, r}}$ is uniformly bounded also in $\varepsilon$ let $\psi$ be in $D_{0}$. Then

$$
\begin{aligned}
\left\|\left(H_{0}+1\right) e^{i t H_{\varepsilon, r}} \psi\right\| & \leqq a\left\|\left(H_{\varepsilon, r}+b\right) e^{i t H_{\varepsilon, r}} \psi\right\| \\
& =a\left\|\left(H_{\varepsilon, r}+b\right) \psi\right\| \leqq a^{\prime}\left\|\left(H_{0}+b^{\prime}\right) \psi\right\| .
\end{aligned}
$$

Since $V_{\varepsilon, r}$ is bounded uniformly in $\varepsilon$ we may choose $a$ and $b$ as well as $a^{\prime}$ and $b^{\prime}$ independent of $\varepsilon$, this shows that $e^{i t H_{\varepsilon, r}}$ is uniformly bounded also in $\varepsilon$ as an operator on $D_{0}$.

Lemma 3. Let $V(\alpha)$ be a real continuous function which tends to zero at infinity. Then $e^{i t H_{\varepsilon, r}}$ converge strongly to $e^{i t H_{0}}$ both as operators on $\mathscr{F}$ and as operators on $D_{0}$. Moreover both convergences are uniform on compact intervals in $t$.

Proof. We have already seen that $e^{i t H_{\varepsilon, r}}$ and $e^{i t H_{0}}$ are strongly continuous semigroups on both $D_{0}$ and $\mathscr{F}$, and they are uniformly bounded both on $D_{0}$ and $\mathscr{F}$ with respect to $\varepsilon$ and $t$. Therefore by the Trotter-Kato semigroup theorem (see Ref. [6], Ch. XI, § 12) it is enough to prove that $\left(z-H_{\varepsilon, r}\right)^{-1}$ converge strongly both on $D_{0}$ and $\mathscr{F}$ to $\left(z-H_{0}\right)^{-1}$ for at least one $z$. Using that $V_{\varepsilon, r}$ is bounded we get for $z$ nonreal or sufficiently negative.

$$
\left(z-H_{\varepsilon, r}\right)^{-1}-\left(z-H_{0}\right)^{-1}=\left(z-H_{\varepsilon, r}\right)^{-1} V_{\varepsilon, r}\left(z-H_{0}\right)^{-1} .
$$


Since $\left(z-H_{\varepsilon, r}\right)^{-1}$ is bounded uniformly in $\varepsilon$, we get from Theorem 1 that $\left(z-H_{\varepsilon, r}\right)^{-1}-\left(z-H_{0}\right)^{-1}$ converge strongly to zero as an operator on $\mathscr{F}$. To see that it also converge strongly as an operator on $D_{0}$, we apply $\left(H_{0}+1\right)$ from the left in (2.7). Since $V_{\varepsilon, r}$ is bounded uniformly in we see that $\left(H_{0}+1\right)\left(z-H_{\varepsilon, r}\right)^{-1}$ is bounded uniformly in $\varepsilon$. So again it follows from Theorem 1 that $\left(z-H_{\varepsilon, r}\right)^{-1}-\left(z-H_{0}\right)^{-1}$ converge strongly to zero as an operator on $D_{0}$.

Theorem 2. Let $V(\alpha)$ be a continuous real function which tends to zero at infinity. Then for $h$ in $L_{2}$ and $\psi$ in $D_{0}$, we have that $\phi_{\varepsilon, r, t}(h) \psi$ converge strongly to $\phi^{t}(h) \psi$ as $\varepsilon$ tends to zero. Moreover, if $V(\alpha)$ has a bounded and uniformly continuous derivative $V^{\prime}(a)$ and $h$ is in $L_{1} \cap L_{2}$, then $\phi_{\varepsilon, r, t}(h)-\phi^{t}(h)$ is a bounded operator which converge strongly to zero as $\varepsilon$ tends to zero.

Proof. Let $\psi$ be in $D_{0}$. Since $H_{0}$ has a strictly positive mass $m$, we know that $\phi(h)$ is a bounded linear map from $D_{0}$ to $\mathscr{F}$. Lemma 3 then gives us that $e^{i t H_{\varepsilon, r}} \psi$ converge strongly to $\phi(h) \psi$. For the moreover part we shall need the following lemma which is the corollary 2 of Ref. [3].

Lemma 4. Let $V(\alpha)$ be a continuous bounded real function with a bounded and uniformly continuous derivative $V^{\prime}(\alpha)$. Then for $h$ in $L_{1} \cap L_{2}$, we have that

$$
\left\|\phi_{\varepsilon, r, t}(h)-\phi^{t}(h)\right\| \leqq C\left(|t|^{3}+1\right)\left\|V^{\prime}\right\|_{\infty}\|h\|_{1},
$$

where $C$ depends only on the mass $m$.

For the proof of this lemma we refer to Ref. [3]. From this lemma we get that $\phi_{\varepsilon, r, t}(h)-\phi^{t}(h)$ is bounded uniformly in $\varepsilon$. We have already proved that it converges strongly to zero on $D_{0}$. Using now the uniform boundedness and the fact that $D_{0}$ is dense in $\mathscr{F}$ we get that it converges strongly to zero on all of $\mathscr{F}$. This completes the proof of Theorem 2.

Remark. The assumption in Theorem 1 that $V(\alpha)$ should tend to zero at infinity was chosen mainly to get a convenient class of functions to work with, and we may prove Theorem 1 for a larger class of bounded continuous real functions. We see that the class of continuous functions which are zero at infinity, arise as the uniform closure of the Fourier transforms of $L_{1}$-functions. The $L_{1}$-functions were introduced in Lemma 1. But we see that Lemma 1 remains true if we assume that $V(\alpha)$ is the Fourier transform of a bounded measure $\mu$ such that $\{0\}$ has $\mu$-measure zero. From this we see that it is enough to assume in Theorem 1 that $V(\alpha)$ is in the uniform closure of the Fourier transforms of bounded measures for which $\{0\}$ is a null set. The almost periodic functions which are orthogonal, in the sense of almost periodic functions, to the constant function, belongs for instance to this class of functions. 


\section{The Vacuum for the Cut-Off-Interaction}

The discussion of the vacuum for the cut-off interaction in this section is mainly an adaption of the discussion of the vacuum for the space cutoff $\lambda \phi^{4}$ interaction in two space time dimensions by Glimm and Jaffé [1]. The fact that $V(\alpha)$ is a bounded function leads to some minor changes from Glimm and Jaffé's discussion.

Let $\mathscr{H}_{l}$ be the subspace of $\mathscr{H}$ consisting of functions which are constant on each cube of length $l$ in $R^{3}$ and with center at the lattice points $\left(\ln _{1}, \ln _{2}, \ln _{3}\right)$ where $n_{1}=0, \pm 1, \pm 2, \ldots$ Let $\tilde{\mathscr{H}}_{l}$ be the orthogonal complement of $\mathscr{H}_{l}$, and let $\mathscr{F}_{l}$ and $\tilde{\mathscr{F}}_{l}$ be the Fock spaces with $\mathscr{H}_{l}$ and $\tilde{\mathscr{H}}_{l}$ as one particle spaces. $\mathscr{F}_{l}$ and $\tilde{\mathscr{F}}_{l}$ are then in a natural way identified with subspaces of $\mathscr{F}$. Let $P_{l}$ be the orthogonal projection onto $\mathscr{F}_{l}$. The direct sum decomposition $\mathscr{H}=\mathscr{H}_{1} \oplus \tilde{\mathscr{H}}_{1}$ gives us the tensor product decomposition $\mathscr{F}=\mathscr{F}_{1} \otimes \tilde{\mathscr{F}}_{l}$. Relative to this tensor produce decomposition, the identification of $\mathscr{F}_{l}$ with a subspace in $\mathscr{F}$ is given by $\mathscr{F}_{l} \otimes \tilde{\Omega}_{l}$, where $\tilde{\Omega}$ is the Fock vacuum in $\tilde{\mathscr{F}}_{l}$; and similar for $\mathscr{F}_{l}$. From the definition of $\phi_{\varepsilon}(x)$ we see that $\phi_{\varepsilon}(x)=a^{*}\left(h_{x}\right)+a\left(\bar{h}_{x}\right)$, where

$$
h_{x}(p)=2^{-\frac{1}{2}}(2 \pi)^{-\frac{3}{2}} e^{-i p x} \hat{g}_{\varepsilon}(p) \text {. }
$$

We now define

$$
\phi_{\varepsilon, l}(x)=a^{*}\left(P_{l} h_{x}\right)+a\left(P_{l} \bar{h}_{x}\right) .
$$

Since $P_{l}$ commutes with complex conjugation we see that $\phi_{\varepsilon, l}(x)$ is self-adjoint. It follows from (4.1) that relative to the decomposition $\mathscr{\mathscr { F }}=\tilde{\mathscr{F}}_{l} \otimes \tilde{\mathscr{F}}_{l}, \phi_{\varepsilon, l}(x)$ takes the form

$$
\phi_{\varepsilon, l}(x)=\phi_{\varepsilon, l}^{(1)}(x) \otimes 1,
$$

where $\phi_{\varepsilon, l}^{(1)}(x)$ is the restriction of $\phi_{\varepsilon, l}(x)$ to $\mathscr{F}_{l}$. We now define

$$
V_{\varepsilon, r, l}=\int_{|x| \leqq r} V\left(\phi_{\varepsilon, l}(x)\right) d x .
$$

From (4.2) we get that

$$
V_{\varepsilon, r, l}=V_{\varepsilon, r, l}^{(1)} \otimes 1
$$

where again $V_{\varepsilon, r, l}^{(1)}$ is the restriction of $V_{\varepsilon, r, l}$ to $\mathscr{F}_{l}$.

$H_{0}$ is uniquely defined by its action as multiplication by $\omega(p)$ in the one particle space $\mathscr{H}$. We define $H_{0, l}$ as the operator we get by substituting $\omega_{l}$ for where $\omega_{l}=P_{l} \omega P_{l}$, i.e. the average of $\omega$ over the cubes. We then see that $H_{0, l}$ commutes with $P_{l}$ and relative to the decomposition $\mathscr{F}=\mathscr{F}_{l} \otimes \tilde{\mathscr{F}}_{l} H_{0, l}$ has the form

$$
H_{0, l}=H_{0, l}^{(1)} \otimes 1+1 \otimes H_{0, l}^{(2)}
$$


where $H_{0, l}^{(1)}$ and $H_{0, l}^{(2)}$ are the restrictions of $H_{0, l}$ to $\mathscr{F}_{l}$ and to $\tilde{\mathscr{F}}_{l}$. We now define

$$
H_{\varepsilon, r, l}=H_{0, l}+V_{\varepsilon, r, l},
$$

and we see that $H_{\varepsilon, r, l}$ are self-adjoint on $D_{0}$.

Lemma 5. Let $V(\alpha)=\int e^{i \alpha s} d \mu(s)$, where $\mu$ is a bounded measure, then for $z$ nonreal or sufficiently negative $\left(z-H_{\varepsilon, r, l}\right)^{-1}$ converge in norm to $\left(z-H_{\varepsilon, r}\right)^{-1}$ as $l$ tends to zero.

Proof. Since $H_{\varepsilon, r}$ and $H_{\varepsilon, r, l}$ has the same domain of definition we get

$$
\left(z-H_{\varepsilon, \boldsymbol{r}, l}\right)^{-1}-\left(z-H_{\varepsilon, \boldsymbol{r}}\right)^{-1}=\left(z-H_{\varepsilon, \boldsymbol{r}, l}\right)^{-1}\left(H_{\varepsilon, \boldsymbol{r}, l}\right)\left(z-H_{\varepsilon, r}\right)^{-1} .
$$

$H_{\varepsilon, r, l}$ is bounded below uniformly in $l$, therefore it is enough to prove that $\left(H_{\varepsilon, r, l}-H_{\varepsilon, r}\right)\left(z-H_{\varepsilon, r}\right)^{-1}$ tends to zero in norm. Since $V_{\varepsilon, r}$ is bounded this is the same as proving that $\left(H_{\varepsilon, r, l}-H_{\varepsilon, r}\right)\left(z-H_{0}\right)^{-1}$ tends to zero in norm. That $\left\|\left(H_{0, l}-H_{0}\right)\left(z-H_{0}\right)^{-1}\right\|$ tends to zero follows from a direct computation with $\omega$ and $\omega_{l}$ (see Ref. 1). To see that $\left(V_{\varepsilon, r, l}-V_{\varepsilon, r}\right)\left(z-H_{0}\right)^{-1}$ tends to zero in norm we have

$$
\begin{aligned}
\|\left(V_{\varepsilon, r, l}-\right. & \left.V_{\varepsilon, r}\right)\left(z-H_{0}\right)^{-1} \| \\
& \leqq \int_{|x|<r} d x \int d|\mu|(s)\left\|\left(e^{i s \phi_{\varepsilon, l}(x)}-e^{i s \phi_{\varepsilon}(x)}\right)\left(z-H_{0}\right)^{-1}\right\| \\
& =\int_{|x|<r} d x \int d|\mu|(s)\left\|\left(e^{\left.i s \phi_{\varepsilon, l}(x)-\phi_{\varepsilon}(x)\right)}-1\right)\left(z-H_{0}\right)^{-1}\right\| .
\end{aligned}
$$

However

$$
e^{i s\left(\phi_{\varepsilon, l}(x)-\phi_{\varepsilon}(x)\right)}-1=\frac{e^{i s\left(\phi_{\varepsilon, l}(x)-\phi_{\varepsilon}(x)\right.}-1}{\phi_{\varepsilon, l}(x)-\phi_{\varepsilon}(x)}\left(\phi_{\varepsilon, l}(x)-\phi_{\varepsilon}(x)\right) .
$$

Since $\frac{e^{i \alpha}-1}{\alpha}$ is a uniformly bounded function on the real axis we therefore get

$$
\begin{aligned}
\left\|\left(V_{\varepsilon, r, l}-V_{\varepsilon, r}\right)\left(z-H_{0}\right)^{-1}\right\| & \leqq C \int_{|x| \leqq r} d x\left\|\left(\phi_{\varepsilon, l}(x)-\phi_{\varepsilon}(x)\right)\left(z-H_{0}\right)^{-1}\right\| \\
& \leqq \frac{2 C}{m} \int_{|x| \leqq r} d x\left\|P_{l} h_{x}-h_{x}\right\|\left\|\left(H_{0}+1\right)\left(z-H_{0}\right)^{-1}\right\|,
\end{aligned}
$$

where we have used the well known estimate

$$
\left\|a^{\#}(h) \psi\right\| \leqq \frac{1}{m}\|h\|\left\|\left(H_{0}+1\right) \psi\right\| .
$$

Since $\left\|P_{l} h_{x}-h_{x}\right\|$ converge to zero the lemma is proved.

Corollary 1. Let $V(\alpha)$ be in the uniform closure of the Fourier transform of bounded measures, then for $z$ non-real or sufficiently negative $\left(z-H_{\varepsilon, r, l}\right)^{-1}$ converge in norm to $\left(z-H_{\varepsilon, r}\right)^{-1}$ as $l$ tends to zero. 
Proof. Since $V(\alpha)$ may be uniformly approximated by a Fourier transform $\tilde{V}(\alpha)$ of a bounded measure, we get that $V_{\varepsilon, r, l}$ is approximated in norm by $\tilde{V}_{\varepsilon, r, l}$ uniformly in $l$. But this gives us that $\left(z-H_{\varepsilon, r, l}\right)^{-1}$ is approximated in norm by $\left(z-\tilde{H}_{\varepsilon, r, l}\right)^{-1}$ uniformly in $l$. The norm convergence of $\left(z-H_{\varepsilon, r, l}\right)^{-1}$ then follows from the norm convergence of $\left(z-\tilde{H}_{\varepsilon, r, l}\right)^{-1}$. This proves the corollary.

From (4.4) and (4.5) we get that relative to the decomposition $\mathscr{F}=\mathscr{F}_{l} \otimes \tilde{\mathscr{F}}_{l}$ we have

$$
H_{\varepsilon, r, l}=H_{\varepsilon, r, l}^{(1)} \otimes 1+1 \otimes H_{0, l}^{(2)}
$$

where $H_{\varepsilon, r, l}^{(1)}$ is the restriction of $H_{\varepsilon, r, l}$ to $\mathscr{F}_{l}$. We now define a vacuum of a semi-bounded operator $H$ as a normalized eigenvector of $H$ with eigenvalue equal to the lower spectral bound of $H$. Since $H_{0, l}^{(1)}$ has a compact resolvent and $V_{\varepsilon, r, l}$ is bounded we see that $H_{\varepsilon, r, l}^{(1)}$ has a compact resolvent and therefore a vacuum $\Omega_{\varepsilon, r, l}$. Since $H_{0, l}^{(2)}$ is positive we get from (4.6) that $\Omega_{\varepsilon, r, l}$ is also a vacuum for $H_{\varepsilon, r, l}$. We now have the following theorem.

Theorem 3. Let $V(\alpha)$ be in the uniform closure of the Fourier transform of bounded measures. Then both $H_{\varepsilon, r, l}$ and $H_{\varepsilon, r}$ have unique vacuums $\Omega_{\varepsilon, r, l}$ and $\Omega_{\varepsilon, r}$. Moreover with the phases determined by $\left(\Omega, \Omega_{\varepsilon, r, l}\right)>0$ and $\left(\Omega, \Omega_{\varepsilon, r}\right)>0$ where $\Omega$ is the Fock vaccum, we have that $\Omega_{\varepsilon, r, l}$ converge strongly to $\Omega_{\varepsilon, r}$ as $l$ tends to zero.

Proof. The proof of the corresponding thing in Glimm and Jaffe [1] goes in two steps. First they prove that any sequence $\Omega_{\varepsilon, r, l_{n}}$ has a subsequence $\Omega_{\varepsilon, r, l_{n}}$ which converge to a vacuum $\Omega_{\varepsilon, r}$ of $H_{\varepsilon, r}$. We see from their proof that once we have Corollary 1 and formula (4.6) then this part of their proof can be carried over. Their second step is to prove that $\Omega_{\varepsilon, r, l}$ and $\Omega_{\varepsilon, r}$ are unique. This part is done with the help of the theory of positive ergodic kernels and carries over point by point to our case; apart from some simplifications due to the fact that $V(\alpha)$ is bounded. For the details we refer to Ref. [1]. This proves Theorem 3.

We shall now be interested in what happens to the vacuum $\Omega_{\varepsilon, r}$ as $\varepsilon$ tends to zero. In view of Theorem 1 we would expect it to converge to the Fock vacuum $\Omega$, if it converges at all.

Theorem 4. Let $V(\alpha)$ be a continuous real function which tends to zero at infinity. If $\left.r^{3} V\right|_{\infty} \leqq C$, where $C$ is positive and depends only on the mass $m$; then with the phases determined as in Theorem $3, \Omega_{\varepsilon, r}$ converge strongly to $\Omega$ as $\varepsilon$ tends to zero.

$$
\text { Proof. } r^{3}\|V\|_{\infty} \leqq C \text { implies that }\left\|V_{\varepsilon, r}\right\| \leqq \frac{4 \pi}{3} C \text {. Since the eigenvalue }
$$

of $H_{0}$ corresponding to the eigenvector $\Omega$, is separated from the rest of the spectrum by a distance equal to $m$, we know by the theory of regular 
perturbation that there is an interval $I \subset\langle 0, m\rangle$ such that the spectrum of the operators $H=H_{0}+V$ with $\|V\| \leqq C^{\prime}$, do not intersect $I$ (see Theorem 4.10, Ch. V, Ref. [5]). Moreover $C^{\prime}$ depends only on $m$; and to the left of $I, H$ has a single eigenvalue which depends analytic on $V$.

If we now choose $C=\frac{3}{4 \pi} C^{\prime}$ we see that zero is a stable eigenvalue under the perturbation $H_{\varepsilon, r}=H_{0}+V_{\varepsilon, r}$. Stable eigenvalues is here used in the sense of Kato (see $\S 1.4$, Ch. VIII, Ref. [5]). Since $V_{\varepsilon, r}$ is uniformly bounded and tends strongly to zero as $\varepsilon$ tends to zero by Theorem 1, we find that $H_{\varepsilon, r}$ converge to $H_{0}$ in the generalized strong sense of Kato and therefore the theory of asymptotic perturbation applies (Ch. VIII, Ref. [5]). Since zero is a stable eigenvalue we therefore get that the projection onto $\Omega_{\varepsilon, r}$ converge in norm to the projection onto $\Omega$. Hence $\Omega_{\varepsilon, r}\left(\Omega_{\varepsilon, r}, \Omega\right)$ converge strongly to $\Omega$, and since the phases are determined as in Theorem 3 this gives us that $\Omega_{\varepsilon, r}$ converge strongly to $\Omega$. This proves the theorem.

\section{The Asymptotic Fields and the Scattering Operator for the Cut-Off Interaction}

Throughout this section we shall assume that $V(\alpha)$ is differentiable with a bounded and uniformly continuous derivative $V^{\prime}(\alpha)$. In Ref. [2], we discussed the asymptotic fields for the cut-off interaction, and we begin this section by stating some of the results obtained in Ref. [2]. The assumption on $V(\alpha)$ in Ref. [2] was that it was the Fourier transform of a bounded measure with a bounded first order moment. However, using the Lemma 1 of Ref. [3] it is easy to see that the results of Ref. [2] holds if we assume that $V(\alpha)$ has a bounded and uniformly continuous derivative $V^{\prime}(\alpha)$.

The interaction picture annihilation-creation operators corresponding to the cut-off interaction is defined by

$$
a_{t}^{\sharp}(h)=e^{-i t H_{\varepsilon, r}} e^{i t H_{0}} a^{\ddagger}(h) e^{-i t H_{0}} e^{i t H_{\varepsilon, r}}
$$

where $h$ is in $\mathscr{H}$. Let $D_{\frac{1}{2}}$ be the domain of $H_{0}^{\frac{1}{2}}$, which is also the domain of $\left(H_{\varepsilon, r}+b\right)^{\frac{1}{2}}$. Then $a_{t}^{\ddagger}(h)$ is a closed operator with domain containing $D_{\frac{1}{2}}$ (see Ref. [2]). Since

$$
e^{i t H_{0}} a^{\sharp}(h) e^{-i t H_{0}}=a^{*}\left(h_{ \pm t}\right),
$$

where + goes with $a^{*}$ and - with $a$ and $h_{t}(p)=e^{i t \omega(p)} h(p)$, we may write (5.1) as

$$
a_{t}^{\ddagger}(h)=e^{-i t H_{\varepsilon, r}} a^{\ddagger}\left(h_{ \pm t}\right) e^{i t H_{\varepsilon, r}} .
$$

The following three theorems are proved in Section 3 of Ref. [2]. 
Theorem 5. Let $h$ be in $\mathscr{H}$ and $\phi$ in $D_{\frac{1}{2}}$. Then $a_{t}^{\sharp}(h) \phi$ converge strongly to $a_{ \pm}^{\#}(h) \phi$ as $t$ tends to $\pm \infty$. The asymptotic limit operators $a_{ \pm}^{\#}(h)$ are closed operators with domain containing $D_{\frac{1}{2}}$, and $a_{ \pm}^{\#}(h)$ is a bounded linear map from $\mathscr{H}$ into the Banach space of bounded linear maps from $D_{\frac{1}{2}}$ into $\mathscr{F}$. Moreover $a_{ \pm}(\bar{h})$ and $a_{ \pm}^{*}(h)$ are adjoints.

Theorem 6. Let $h$ and $g$ be in $\mathscr{H}$. Then $a_{ \pm}^{\#}(h)$ maps $D_{0}$ into the domain of $a_{ \pm}^{\#}(g)$, and $a_{ \pm}^{\#}(g) a_{ \pm}^{\#}(h)$ is a bounded linear map from $\mathscr{H} \otimes \mathscr{H}$ into the Banach space of bounded linear maps from $D_{0}$ into $\mathscr{F}$. Moreover $a_{ \pm}^{\#}(h)$ satisfy the same commutation relations on $D_{0}$ as do $a^{\sharp}(h)$ on $H_{0} . H_{\varepsilon, r}$ and $a_{ \pm}^{\#}(h)$ satisfy the same commutation relations as do $H_{0}$ and $a^{\sharp}(h)$, in the sense that on $D_{\frac{1}{2}}$

$$
\begin{aligned}
& e^{i t H_{\varepsilon, r}} a_{ \pm}(h) e^{-i t H_{\varepsilon, r}}=a_{ \pm}\left(h_{-t}\right), \\
& e^{i t H_{\varepsilon, r}} a_{ \pm}^{*}(h) e^{-i t H_{\varepsilon, r}}=a_{ \pm}^{*}\left(h_{-t}\right) .
\end{aligned}
$$

Theorem 7. Let $\Phi$ be an eigenvector of $H_{\varepsilon, r}$. Then for any $h$ in $\mathscr{H}$

$$
a_{ \pm}(h) \Phi=0 .
$$

For the proof of these theorems see Ref. [2].

In Section 4 of Ref. [2] we show that any vector that is annihilated by $a_{ \pm}(h)$ for all $h$ in $\mathscr{H}$, is in the domain of $a_{ \pm}^{*}\left(\mathrm{~h}_{n}\right)$ for all $h_{1}, \ldots, h_{n}$ in $\mathscr{H}$ and all $n$. Since the vacuum $\Omega_{\varepsilon, r}$ is an eigenvector of $H_{\varepsilon, r}$ we get by Theorem 5.3 that it is in the domain of $a_{ \pm}^{*}\left(h_{1}\right) \ldots a_{ \pm}^{*}\left(h_{n}\right)$. Let $\mathscr{\mathscr { F }}_{ \pm}$be the smallest closed subspace of $\mathscr{F}$ containing all vectors of the form $a_{ \pm}^{*}\left(h_{1}\right) \ldots a_{ \pm}^{*}\left(h_{n}\right) \Omega_{\varepsilon, r}$. Due to the commutation relations for $a_{ \pm}^{\ddagger}(h)$, we see that $\mathscr{F}_{ \pm}$are Fock spaces with annihilation-creation operators $a_{ \pm}^{\sharp}$ and vacuum $\Omega_{\varepsilon, r}$. By regarding $\mathscr{F}_{ \pm}$as Fock spaces in this way we get a natural identification of the asymptotic Fock spaces $\mathscr{F}_{ \pm}$with the free Fock space $\mathscr{F}$ given by the "wave" operators $W_{ \pm}$, where $W_{ \pm}$are defined by

$$
W_{ \pm} a^{*}\left(h_{1}\right) \ldots a^{*}\left(h_{n}\right) \Omega=a_{ \pm}^{*}\left(h_{1}\right) \ldots a_{ \pm}^{*}\left(h_{n}\right) \Omega_{\varepsilon, r} .
$$

Due to the commutation relations for $a_{ \pm}^{\mp}$ we get that $W_{ \pm}$are isometric from $\mathscr{F}$ onto $\mathscr{F}_{ \pm}$. We have already seen that the vacuum $\Omega_{\varepsilon, r}$ is unique. It is therefore natural to identify $\mathrm{a}_{ \pm}^{*}\left(h_{1}\right) \ldots \Omega_{\varepsilon, r}$ with an outgoing (incoming) $n$-particle state with momentum distribution given by $h_{1}, \ldots, h_{n}$, for the cut-off interaction.

The scattering amplitude for the cut-off interaction for $m$ incoming particles with momentum distribution $h_{1}, \ldots, h_{m}$; and $n$ outgoing particles with momentum distribution $g_{1}, \ldots, g_{n}$ is then given by

$$
\left(a_{+}^{*}\left(g_{1}\right) \ldots a_{+}^{*}\left(g_{n}\right) \Omega_{\varepsilon, r}, a_{-}^{*}\left(h_{1}\right) \ldots a_{-}^{*}\left(h_{m}\right) \Omega_{\varepsilon, r}\right) .
$$


By (5.4) we get that this is equal to

$$
\begin{aligned}
& \left(W_{+} a^{*}\left(g_{1}\right) \ldots a^{*}\left(g_{n}\right) \Omega, W_{-} a^{*}\left(h_{1}\right) \ldots a^{*}\left(h_{m}\right) \Omega\right) \\
& \quad=\left(a^{*}\left(g_{1}\right) \ldots a^{*}\left(g_{n}\right) \Omega, W_{+} W_{-} a^{*}\left(h_{1}\right) \ldots a^{*}\left(h_{m}\right) \Omega\right) .
\end{aligned}
$$

Hence we get that the scattering operator for the cut-off interaction is given by

$$
S=W_{+}^{*} W_{-} .
$$

Since $W_{ \pm}$are isometrics in $\mathscr{F}$ we see that $\|S\| \leqq 1$, and that $S$ is unitary if and only if $\mathscr{F}_{+}=\mathscr{F}_{-}$. Let $E_{\varepsilon, r}$ be the eigenvalue of $\Omega_{\varepsilon, r}$. From the commutation relations for $a_{ \pm}^{\sharp}$ and $H_{\varepsilon, r}$ we get from (5.4) that

$$
\left(H_{\varepsilon, r}-E_{\varepsilon, r}\right) W_{ \pm}=W_{ \pm} H_{0}
$$

and this together with (5.7) gives us that

$$
H_{0} S=S H_{0} .
$$

We shall now be interested in what happens with $S$ if we keep $V(\alpha)$ and $r$ fixed but let $\varepsilon$ tend to zero.

Lemma 6. Let $h$ be in $\mathscr{H}$. Then $V_{\varepsilon, r}$ leaves the domain of $a^{\sharp}(h)$ invariant and

$$
\left\|\left[a^{\sharp}(h), V_{\varepsilon, r}\right]\right\| \leqq C \cdot \sup \left|\int h(p) \hat{g}_{\varepsilon}(p)(p)^{-\frac{1}{2}} e^{i x p} d p\right|,
$$

where $C$ depends only on $r$ and on $\left|V^{\prime}\right|_{\infty}$. Moreover

$$
a_{t}^{\sharp}(h)-a^{\sharp}(h)=i \int_{0}^{t} e^{-i s H_{\varepsilon, r}}\left[a^{\sharp}\left(h_{ \pm s}\right), V_{\varepsilon, r}\right] e^{i s H_{\varepsilon, r}} d s,
$$

where the integral is taken in the strong sense.

For the proof of this lemma see Section 3, Ref. [2]. In Ref. [2] we assume that $V(\alpha)$ is the Fourier transform of a bounded measure with a bounded first order moment. The technique for the proof when $V(\alpha)$ has a bounded uniformly continuous derivative $V^{\prime}(\alpha)$ is to be found in the proof of Lemma 1, Ref. [3].

Let $\mathscr{H}_{0}$ be the set of functions in $\mathscr{H}$ which has compact support and is zero in a neighborhood of zero. Let $h$ be in $\mathscr{H}_{0}$. From Lemma 6 we then see that $\left\|\left[a^{\sharp}\left(h_{ \pm s}\right), V_{\varepsilon, r}\right]\right\|$ converge to zero faster than any inverse power in $|s|$, and the convergence is uniform in $\varepsilon$. From Lemma 6 we also get that

$$
\left\|a_{+}^{\sharp}(h)-a_{t}^{\sharp}(h)\right\| \leqq \int_{t}^{\infty}\left\|\left[a^{\sharp}\left(h_{ \pm s}\right), V_{\varepsilon, r}\right]\right\| d s .
$$

Hence for any $\delta>0$ we may choose $t$ so large that $\left\|a_{+}^{\sharp}(h)-a_{t}^{\sharp}(h)\right\|<\delta$, and this choice of $t$ may be done independently of $\varepsilon$. 
Lemma 7. Assume also that $V(\alpha)$ tends to zero at infinity. Then for $h$ in $\mathscr{H}_{0}, a_{ \pm}^{\sharp}(h)-a^{\sharp}(h)$ is norm bounded uniformly in $\varepsilon$ and converge strongly to zero as \& tends to zero.

Proof. The uniform norm boundedness follows from Lemma 6. To prove the strong convergence write

$$
a_{+}^{\sharp}(h)-a^{\sharp}(h)=\left(a_{+}^{\ddagger}(h)-a_{t}^{\ddagger}(h)\right)+\left(a_{t}^{\sharp}(h)-a^{\sharp}(h)\right) .
$$

We have already seen that the first term can be made smaller than $\delta$ and the choice of $t$ does not depend on $\varepsilon$. By Lemma 3 the last term tends strongly to zero on $D_{0}$. Hence we get that $a_{+}^{\sharp}(h)-a^{\sharp}(h)$ converge strongly to zero on $D_{0}$. The uniform boundedness then gives us strong convergence on all of $\mathscr{F}$. This proves the lemma.

Theorem 8. Let $V(\alpha)$ be a differentiable real function which tends to zero at infinity, and assume that $V^{\prime}(\alpha)$ is bounded and uniformly continuous. If $r^{3}\|V\|_{\infty} \leqq C$, where $C$ is the constant of Theorem 4 , then the scattering operator $S$ converges weakly to 1 as $\varepsilon$ tends to zero.

Proof. From Theorem 4 we get that $\Omega_{\varepsilon, r}$ converge strongly to $\Omega$ as $\varepsilon$ tends to zero. Let $h$ be in $\mathscr{H}_{0}$. From Lemma 7 we then see that $a^{*}(h) \Omega_{\varepsilon, r}$ converge strongly to $a^{*}(h) \Omega$, since

$$
a_{ \pm}^{*}(h) \Omega_{\varepsilon, r}-a^{*}(h) \Omega=\left(a_{+}^{*}(h)-a(h)\right) \Omega_{\varepsilon, r}+a^{*}(h)\left(\Omega_{\varepsilon, r}-\Omega\right) .
$$

In the same way we see that for $h_{1}, \ldots, h_{n}$ in $\mathscr{H}_{0} a_{ \pm}^{*}\left(h_{1}\right) \ldots a_{ \pm}^{*}\left(h_{n}\right) \Omega_{\varepsilon, r}$ $=W_{ \pm} a^{*}\left(h_{1}\right) \ldots a^{*}\left(h_{n}\right) \Omega$ converge strongly to $a^{*}\left(h_{1}\right) \ldots a^{*}\left(h_{n}\right) \Omega$. Hence $W_{ \pm}$converge strongly to 1 on a dense subset. Since $W_{ \pm}$are isometries we conclude that they converge strongly to 1 on $\mathscr{F}$. It follows then from (5.7) that $S$ converges weakly to 1 . This proves the theorem.

Remark. From the definition of the scattering operator we see that if $V_{1}(\alpha)-V_{2}(\alpha)=$ constant then the cut-off interaction corresponding to $V_{1}(\alpha)$ and $V_{2}(\alpha)$ has the same scattering operator. This fact of course extends the validity of Theorem 8 somewhat.

\section{Removal of the Cut-Off, and the Scattering Operators for Scalar Fields}

In the last section we proved that the scattering operator $S_{\varepsilon, r}(V)$ existed for the cut-off interaction $\int_{|x|<r} V\left(\phi_{\varepsilon}(x)\right) d x$, under the assumption that $V(\alpha)$ has a bounded and uniformly continuous derivative $V^{\prime}(\alpha)$. Since $\left\|S_{\varepsilon, r}(V)\right\| \leqq 1$ for all $\varepsilon, r$ and $V$, we may use the fact that the unit ball in $\mathscr{F}$ is weakly compact to construct a scattering operator $S_{\varepsilon, r}(F)$ for a larger class of cut-off interactions $\int_{|x| \leqq r} F\left(\phi_{\varepsilon}(x)\right) d x$. 
Let $F(\alpha)$ be any continuous function of $\alpha$, and let $V_{n}(\alpha)$ be a sequence of functions with bounded and uniformly continuous derivatives such that $V_{n}(\alpha)$ converge pointwise to $F(\alpha)$. We know that such a sequence always exists. Since $\left\|S_{\varepsilon, r}\left(V_{n}\right)\right\| \leqq 1$ there is a subsequence $V_{m}(\alpha)$ such that $S_{\varepsilon, r}\left(V_{m}\right)$ converges weakly to an operator $S_{\varepsilon, r}(F)$. $S_{\varepsilon, r}(F)$ may not be unique since it may depend on the sequence $V_{n}(\alpha)$ and on the subsequence $V_{m}(\alpha)$ we choose. But from (5.9) we will always have that $S_{\varepsilon, r}(F)$ commutes with $H_{0}$, and since the unit ball is weakly closed we have also that $\left\|S_{\varepsilon, r}(F)\right\| \leqq 1$.

Having constructed in this way for each $\varepsilon$ and $r$ and $S_{\varepsilon, r}(F)$, we may again use weak compactness to select sequences $\varepsilon_{n}$ tending to zero and $r_{n}$ tending to infinity such that $S_{\varepsilon_{n}, r_{n}}(F)$ converge weakly to a limit $S(F)$. $S(F)$ will commute with $H_{0}$ and $\|S(F)\| \leqq 1$. It is natural to interpret $S(F)$ constructed in this way as the relativistic scattering operator corresponding to the interaction density $F(\phi(x))$. It is probable from what we have seen in the previous sections that $S(F)$ is the identity of $F(\alpha)$ is bounded and tend to zero at infinity even though we have not been able to prove this.

A more general class of scattering operator are obtained by using renormalized interaction densities. A renormalized interaction density is given by a family of continuous functions $F^{\varepsilon}(\alpha)$. As the scattering operator for the renormalized interaction we take a weak limit point of $S_{\varepsilon, r}\left(F^{\varepsilon}\right)$ as $\varepsilon$ tends to zero and $r$ to infinity. As an example consider the $\phi^{4}$ theory. Here

$$
F^{\varepsilon}(\alpha)=a_{0}(\varepsilon) \alpha^{4}+a_{1}(\varepsilon) \alpha^{3}+a_{2}(\varepsilon) \alpha^{2}+a_{3}(\varepsilon) \alpha^{1}
$$

where $a_{i}(\varepsilon)$ will be functions depending on $\varepsilon$. A weak limit point of $S_{\varepsilon, r}\left(F^{\varepsilon}\right)$ as $\varepsilon$ tends to zero and $r$ to infinity is then a scattering operator for the $\phi^{4}$ theory.

We may also introduce the set $\mathscr{S}$ of all local relativistic scattering operators in the following way. Let $\mathscr{S}_{\varepsilon, r}$ be the weak closure of the set $\left\{S_{\tilde{\varepsilon}, \tilde{r}}(V) ; \tilde{\varepsilon} \leqq \varepsilon, \tilde{r} \geqq r\right.$ and $V$ real with a bounded and uniformly continuous derivative $\}$. We then define

$$
\mathscr{S}=\bigcap_{\varepsilon, r} \mathscr{S}_{\varepsilon, r},
$$

$\mathscr{S}$ is then a closed non-empty subset of the unit ball of operators in $\mathscr{F}$. Due to (5.9) all the elements in $\mathscr{S}$ commutes with $H_{0}$. An element $S$ in $\mathscr{S}$ is a local relativistic scattering operator corresponding to a generalized renormalization scheme, in the sense that $S$ would be a weak limit point of a sequence $S_{\varepsilon_{n}, r_{n}}\left(V^{\varepsilon_{n}, r_{n}}\right)$, where the functions $V^{\varepsilon, r}(\alpha)$ may depend in an arbitrary manner on $\varepsilon$ and $r$. It is natural to identify the set $\mathscr{S}$ with the set of all local relativistic scattering operators for scalar fields. 


\section{References}

1. Glimm, J., Jaffé, A.: A $\lambda \phi^{4}$ field theory without cut-offs. II. Annals of Math. 91, No. 2, $362(1970)$

2. Höegh-Krohn, R.: Boson fields under a general class of cut-off interactions. Commun. Math. Phys. 12, 216-225 (1969).

3. - Boson fields under a general class of local relativistic invariant interactions. Commun. Math. Phys. 14, 171-184 (1969).

4. - Asymptotic fields in some models of quantum field theory III. J. Math. Phys. 11, 185-189 (1970).

5. Kato, T.: Perturbation theory for linear operators. Berlin-Heidelberg-New York: Springer 1966.

6. Yosida, K.: Functional analysis. Berlin-Heidelberg-New York: Springer 1965.

Raphael Höegh-Krohn

Joseph Henry Laboratories

Princeton University

Princeton, N. J., 08540 USA 